\title{
Extinction of a taste aversion in the absence of the consummatory response
}

\author{
ROBERT T. BROWN \\ University of North Carolina at Wilmington, Wilmington, North Carolina 28401 \\ R. IAN STEWART \\ University of Glasgow, Glasgow, Scotland \\ and \\ TERRY L. HALL \\ Indiana University and Purdue University at Indianapolis, Indianapolis, Indiana 46204
}

\begin{abstract}
Rats were initially conditioned to avoid saccharin presented orally and were then given 40 intravenous injections of saccharin or saline. Those injected with saccharin showed more rapid extinction of the aversion to saccharin presented orally. Results are related to studies of shock avoidance using curarized subjects.
\end{abstract}

Active avoidance of shock can be influenced by manipulations of the CS made while the subjects are curarized and unable to respond instrumentally. After training dogs to respond to a light to avoid shock, Solomon and Turner (1962) curarized them and presented one tone paired with shock $(\mathrm{S}+)$ and another not paired $(\mathrm{S}-)$. The dogs subsequently emitted the instrumental avoidance response to $S+$ and not to $\mathrm{S}-$, although $\mathrm{S}+$ had been paired with shock only in the absence of any instrumental response. Similarly, extinction of a shockavoidance response can be accelerated if the CS is presented without shock to curarized subjects (Black, 1958; Solomon, Kamin, \& Wynne, 1953). In Black's studies, dogs which had learned an avoidance response to a CS were given either the CS a large number of times while curarized or normal extinction trials. The dogs presented with the CS while curarized extinguished faster.

Analogously, conditioned taste aversions can be conditioned and extinguished when the flavored stimulus is presented in a way that bypasses the subjects' normal consummatory-response system. Rats given an intravenous injection of saccharin solution and then made ill avoided saccharin when first allowed to drink it by mouth (Bradley \& Mistretta, 1971). Domjan and Wilson (1972) either wiped the tonges of curarized rats with saccharin solution or washed saccharin solution through rats' mouths too quickly to permit ingestion, and then induced visceral upset. Subsequently, the rats

This research was conducted when the authors were at the University of North Carolina at Chapel Hill and was supported by the University of North Carolina at Chapel Hill Research Council Grant VC 377. avoided saccharin presented orally. Recently, extinction of a conditioned taste aversion has been induced by direct presentation of saccharin. Baum, Foidart, and Lapointe (1974) conditioned rats to avoid a cyclamate-saccharin solution presented orally and then gave them a single intraperitoneal injection of either a highly concentrated solution of cyclamate-saccharin or water. Rats injected with the sweet solution later drank more cyclamate-saccharin by mouth, demonstrating "flooding," in Baum's terminology.

In the present experiment, Bradley and Mistretta's (1971) technique of intravenous injections of a small low-concentration dose of saccharin was used to accelerate extinction of an orally conditioned taste aversion. A preliminary study had confirmed that an aversion could be conditioned with intravenous saccharin injections as the CS.

\section{METHOD}

\section{Subjects}

The subjects were 20 naive male albino Sprague-Dawley rats, approximately 100 days old at the beginning of the experiment. The rats were housed individually in standard laboratory cages with Purina Lab Chow freely available. Fluids were available on schedules described below.

\section{Procedure}

Conditioning. The rats were initially adapted to a 23-h fluid-deprivation schedule in which water was presented $1 \mathrm{~h}$ at the same time each day. Following 14 days of adaptation, the rats were randomly divided into an experimental (E) group of 13 and a control (C) group of 7. Two conditioning trials were given, 3 days apart. In each conditioning trial, the rats were given $1-\mathrm{h}$ access to $.125 \%$ saccharin solution. Fifteen minutes later, $\mathrm{E}$ rats were intubated with $1 \mathrm{cc}$ of $.12 \mathrm{M}$ lithium chloride for each $30 \mathrm{~g}$ of body weight; 
C rats were intubated with equivalent amounts of ssotons saline. For intubation. an intragastric tube was inserted through the rat's mouth into the stomach, and the flund inlected through an attached syrnge. Three days after the second conditioning tral, one preference test between $.125 \%$ saccharm solution and water was given. Water and saccharin were presented simultaneously in $50-\mathrm{ml}$ luhes. The position of the tubes was reversed after $15 \mathrm{~m} 1 \mathrm{n}$ to control for position preferences. (Rats in previous studies had drunk about half of their consumption in 1 -h lests after $15 \mathrm{~mm}$ ).

Extinction The experimental rats were divided into an experimental IV (intravenous) extinction (EE) group of six and a control IV (CE) group of seven, matched on the bass of saccharin preference on the two-botle lest.

Four series of 10 daily $1-m 1$ intravenous inlections were given in extunction. The FE rats and the unconditoned control (C) rats were infected in the tall ven with 1 of of $154 \mathrm{M}$ saccharin, and the CE rats were mected with 1 co of solomic saline After each injection, the tails were washed to prevent licking of the saccharm solution The $.154 \mathrm{M}$ concentration was that found by Bradley and Mistretta (1971) to be most elfective in conditioning with IV saccharin. Following each serves of injections, one iwo-bottle chorce test between . $1250 \%$ accharm colution and water was grven There was at least 1 week between serles to allow the rals taks to recover from msus damage from the iniections

As little increase in accharm consumpton had occurred after Inlection serien 3, two single-bottle test of accharm make were given Since two-bottle tent are more semstese in delecting conditioning (crote \& Brown, 1971), It was felt that a onc-hottle test, which forces consumption of the possoned flund, would be a more sensitive medsure of extunction (Bn each of these tests. the rats were given a bottle of $125 \%$ saccharin for $1 \mathrm{~h}$

Following the two one-bottle tests, the fourth series ot saccharm injections was given. The study was then terminated because the tals of several rats did not heal dfter the fourth sertes and appeared to have been permanently damaged

Fluid consumption was determined by weighing the tuber on a chemical balance before and after the test Saccharin preference was compuled by the formula (saccharm intake)/(saccharm intake + water intake)

\section{RESULTS}

\section{Conditioning}

As shown in the left part of Figure 1, a strong aversion 10 saccharin was conditioned in the experimental rats. There was no overlap in saccharin preference or intake between the two groups.

\section{Extinction}

There was no apparent effect of the first three series of injections on the rats' preference for saccharin, as shown in the right part of Figure 1. Both groups still showed a strong aversion to saccharin.

The saccharin injections were accelerating extinction of the saccharin aversion in the EE rats, however. This effect was reflected in the two one-bottle tests. The mean saccharin intake by the three groups on the two one-bottle tests is shown in Figure 2. The difference among the group was highly significant $(\mathrm{F}=31.7$, df $-2 / 17, \mathrm{p}<001)$. Subsequent parred comparsons indicated that the intake by the $\mathrm{EL}$, rats was indeed semiticantly

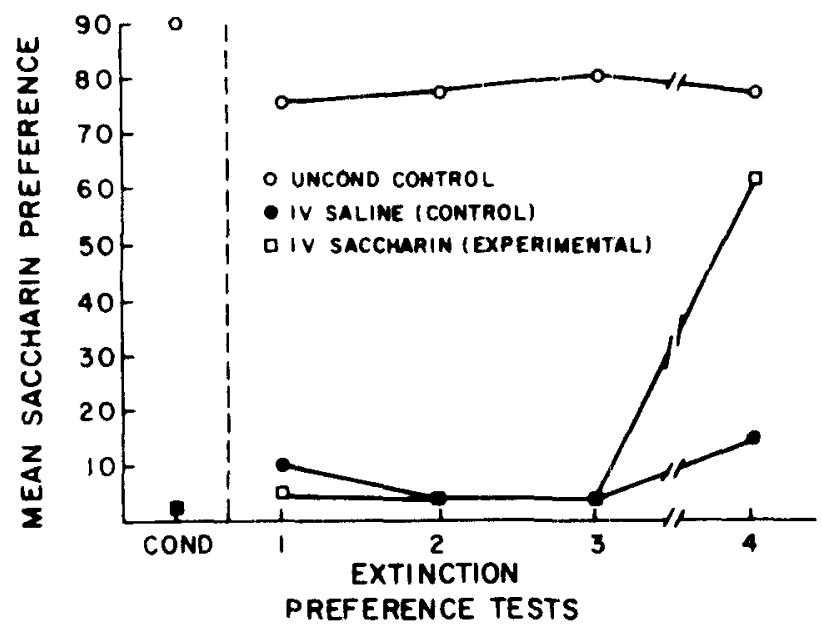

Figure 1. Mean saccharin preferences in two-bottle tests after conditioning (left) and each series of intravenous injections in extinction (right). The break between Preference Tests 3 and 4 in extinction indicates the occurrence of the two one-bottle tests of saccharin intake. (See text for explanation.)

greater than that by the ( $\mathrm{E}$, rats $(t=2.54, \mathrm{p}<.05)$, although less than that by the ( rats $11-5.25$, $n<.(0) 1)$.

()n the basis of the results of the one-bottle tests, a fourth series of IV injections and twobottle tests was given. As Figure 1 shows, the saccharin preference of the EE rats on rest 4 was three times that of the C $I$ rats and approached that of the $G$ rats, confirming that the IV saccharin injection accelerated extinction of the aversion. Further, accharin intake by the $\mathrm{E}$ rats was $20.0 \mathrm{~g}$, whereas that of the $C$ rats was only $4.0 \mathrm{~g}$. Both the EE and CE rats' preferences were above those on the previous tests, suggesting that the one-bottle tests themselves, each of which was functionally an extinction trial, may also have increased extunction. The greater increase in

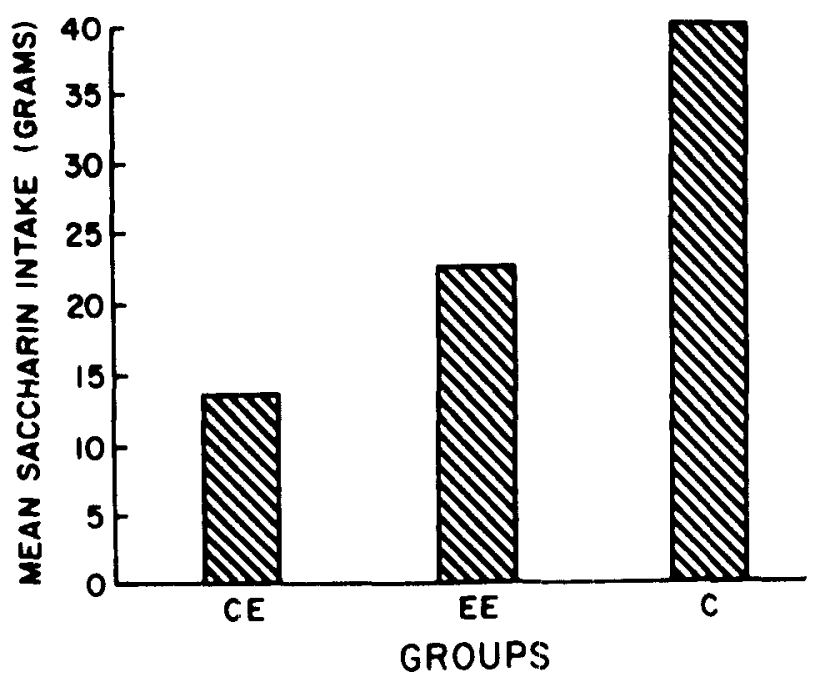

Figure 2. Mean saccharin intake across the two one-bottle tests of saccharin intake given after the third series of intravenous injections in extinction. 
saccharin intake and preference by the $\mathrm{E}$ rats indicates, however, that the one-bottle tests were not responsible themselves for the difference in the final two-bottle test.

Saccharin preferences by the three groups on the four two-bottle tests were analyzed with a multivariate analysis of variance for a repeated-measure design. For this analysis, the means across the four tests and difference scores between the tests of each subject were used to form new and independent measures.

The difference among the three groups across tests on saccharin preference was highly significant $(\mathrm{F}=\mathrm{i} 30.6, \mathrm{df}=2 / 17, \mathrm{p}<.001)$. On the basis of these differences, the preferences of the $\mathrm{EE}$ and $\mathrm{CE}$ rats were compared. Across the four tests, the saccharin preference of the EE rats was significantly higher than that of the $C E$ rats $(\mathrm{F}=6.76, \mathrm{df}=1 / 11, \mathrm{p}<.025)$. As Figure 1 indicates, saccharin preference increased across tests for both groups, but tests did not interact with groups.

\section{DISCUSSION}

Intravenous injections of saccharin accelerated extinction, indicating that a conditioned taste aversion may extinguish in the absence of the consummatory response. The result confirms and extends that of Baum et al. (1974), who used a single, highly intense intraperitoneal injection of the CS fluid to influence extinction.

The large number of injections required before extinction was shown in the preference tests may be a function of several factors. Although 40 injections of saccharin were given, the total amount of fluid presented $(40 \mathrm{cc})$ was small relative to that frequently drunk by rats before they begin to recover from a conditioned taste aversion. Similarly, many oral presentations of saccharin may be required for extinction of a conditioned taste aversion (e.g., Grote \& Brown, 1973). Further, the greater extinction shown by the experimental rats in the singlebottle tests than in the previous two-bottle tests suggests that two-bottle tests are not a sensitive measure of extinction. In two-bottle tests, the subject may completely avoid a fluid for which it has only a slight aversion and still reduce thirst by drinking the other fluid. Finally, the slow extinction may in part result from a generalization decrement in the transfer from intravenous saccharin injections to orally presented saccharin (Domjan, 1973).

In any case, both acquisition and extinction of a taste aversion can occur when the instrumental approach and licking responses are bypassed and no taste stimulus is ingested through normal pathways.
Thus, the taste stimulus may acquire or lose aversive qualities when presented directly to the subjects. In taste aversions, then, direct manipulations of the classically conditioned link between the flavored stimulus (CS) and the malaise-inducing stimulus (UCS) in the absence of the instrumental approach and licking responses associated with normal ingestion can either increase or decrease those responses.

In their analysis of two-process theory of avoidance learning, Rescorla and Solomon (1967) suggested that studies showing that instrumental responses can be mediated by independent classical-conditioning procedures provide strong evidence for a twoprocess explanation. The demonstration that classical-conditioning procedures can modify the instrumental components of taste aversions supports a two-process interpretation of such aversions. Faster extinction of a taste aversion under high deprivation than under low (Grote \& Brown, 1973) also supports this interpretation. High deprivation forces exposure to the aversive CS in the absence of the UCS, accelerating extinction of the classically conditioned link. It should be pointed out that these experiments may also be explained by Bolles' recent expectancy model (1972). In fact, it appears difficult to differentiate experimentally between the two explanations.

The results of the present study and those of Bradley and Mistretta (1971), Domjan and Wilson (1972), and Baum et al. (1974) parallel those in which instrumental avoidance responses increase or decrease as a function of classical-conditioning manipulations made while the subjects were curarized (Black, 1958; Solomon et al., 1953; Solomon \& Turner, 1962). Further, Revusky (1971) has documented through his own and others' work that a number of phenomena originally studied in more traditional learning situations also occur in conditioned taste aversions. These include blocking, overshadowing, and latent inhibition. The question asked by Mackintosh (1973, p. 94) may be usefully reiterated: "How far do the phenomena associated with avoidance of poisonous foods by rats really require the postulation of new principles of learning?"

\section{REFERENCES}

Baum, M. Foidart, D. S., \& Lapointe, A. Rapid extinction of a conditioned taste aversion following unreinforced intraperitoneal injection of the fluid CS. Physiology and Behavior, 1974, 12. $871-873$.

BLACK, A. H. The extinction of avoidance responses under curare. Journal of Comparative and Physiological Psychology, 1958, 51. 519-524.

Bolles, R. C. Reinforcement, expectancy, and learning. Psychological Revie'w. 1972, 79, 394-409. 
Bradley, R. M., \& Mistretta, C. M. Intravascular taste in rats as demonstrated by conditioned aversion to sodium saccharin. Journal of Comparative and Physiological Psychology. 1971. 75, $186-189$.

Domjan, $M$ Role of ingestion in odor-toxicosis learning in the rats Joumal of Comparative and Physiological Psychology, 1973, 84, 507.521.

Domjan, M.. \& W ILson, N. E. Contribution of ingestive behaviors to taste-aversion learning in the rat. Journal of Comparative and Physiological Psychology, 1972, 80, 403-412.

Grote, F. W., \& Brown, R. T. Conditioned taste aversions: Two-stimulus tests are more sensitive than one-stimulus tests. Behavior Research Methods \& Instrumentation, 1971. 3, 311-312.

Grote, F. W., \& Brown, R. T. Deprivation level affects extinction of a conditioned taste aversion. Learning and Motivation, 1973, 4. 314-319.

Mackintosh, N. J. Stimulus selection: Learning to ignore stimuli that predict no change in reinforcement. In R. A. Hinde \& J.
Stevenson-Hinde (Eds). Constramts on larning. New York: Academic Press, 1973.

Rescorla, R. A., \& SOLOMON, R. L. Tuo-process learning theory: Relationships between Pavlovian conditioning and instrumental learning. Psychologtcal Review, 1967, 74, 151-182.

RevUsky. S. The role of interference in association over a delay In W. Honis \& H. James (Eds.), Antmal memory. New York: Academic Press. 1971

Solomon, R. L.. Kamin L. J.. \& Wynne, L C. Traumatic avordance learning The outcomes of several extinction procedures with dogs. Journal of Abnormal and Social Psychology, 1953, 48, 291-303.

Solomon, R L., \& TuRner, L. H. Discriminative classical conditioning in dogs paralyzed by curare can later control discrimmative avoudance responses in the normal state. Psichological Review. 1962, 69. 202-219.

(Received for publication March 3, 1975; revision accepted December 2, 1975.) 